\title{
Stigma against HIV/AIDS among female sex workers and general migrant women in eastern China
}

\author{
Ying Yang ${ }^{1,2 \dagger}$, Jun Wang ${ }^{1 \dagger}$, Feifei Lin', Tao Zhang ${ }^{1}$, Feng Yu², Yanping Zhao ${ }^{2}$ and Tiejun Zhang ${ }^{1 *}$
}

\begin{abstract}
Background: HIV related stigma is a recognized barrier to early detection of HIV and causes great suffering for those affected. However, data regarding HIV related stigma among female sex workers (FSW) in China was limited, with none for comparison between FSW and general migrant women (GMW). Therefore, the aim of this study was to examine HIV related stigma among FSW and GMW in Shanghai, China.

Methods: A community based cross-sectional study with face-to-face interviews was conducted in Shanghai (September 2011 through December 2012), using a structured questionnaire.HIV related stigma scores were examined graphically using boxplot. A logistic regression analysis with the proportional odds model was employed to identify factors affecting HIV related stigma scores.
\end{abstract}

Results: A total of 1,396 subjects, including 721 FSW and 675 GMW, were recruited in the present study. Both groups had substantial misconceptions about HIV/AIDS, although FSW had slightly higher scores on average. Both groups showed a medium level of HIV related stigma (38.34 \pm 6.21 and $38.35 \pm 6.86$ for FSW and GMW, respectively). For the FSW, higher levels of stigma were observed for those who were in the older age groups (age 26-35 years, OR, 2.06, 95\% Cl 1.06-4.01), those who were married ( $\mathrm{OR}, 1.62,95 \% \mathrm{Cl} 1.03-2.54)$, and those who were working at lower-level sex service sites (OR, 1.60, 95\% Cl 1.06-2.43). Conversely, HIV knowledge was inversely associated with the level of HIV related stigma ( $\mathrm{OR}, 0.93,95 \% \mathrm{Cl}$ 0.87-0.98).Among GMW participating in the study, those age in the 26-35 years were more likely to show higher level of stigma (OR, 2.61,95\% CI 1.03-2.54), and HIV knowledge was found to be inversely associated with the HIV related stigma level as well (OR, 0.89, 95\% Cl 0.84-0.95).

Conclusions: The present study suggests that there is an urgent need for the development of appropriate education strategies to reduce HIV related stigma among FSW and GMW in Shanghai, China. In particular, older women, less educated women, and women that have lived in Shanghai a relatively long time should be targeted in future stigma reduction programs.

\section{Background}

HIV-related stigma refers to "prejudice, discounting, discrediting directed at people perceived to have HIV/ AIDS, as well as individuals, groups and communities with which they are associated" [1]. It has a profound effect on people's decisions and behaviors, and can reduce people's willingness to engage in HIV testing, treatment,

\footnotetext{
* Correspondence: tjzhang@shmu.edu.cn

${ }^{\dagger}$ Equal contributors

'Department of Epidemiology, School of Public Health, Fudan University, Shanghai, China and Key Laboratory of Public Health Safety (Fudan University), Ministry of Education, Shanghai, China

Full list of author information is available at the end of the article
}

and prevention. Since the beginning of the HIV/AIDS epidemic over 30 years ago, stigma has been a barrier to HIV testing, diagnosis, treatment and care [2-5]. Although the Chinese government has been putting an enormous effort into the HIV prevention for decades [6], stigmatization against people living with HIV/AIDS (PLWHA) is still perpetuated nationwide [5,7-10].

China has experienced a rapid increase in the incidence of HIV. Annual reports indicate a steady increase in new HIV infections over the past two to three decades $[11,12]$. According to the Joint United Nations Programme on HIV/AIDS (UNAIDS) 2010 report on the global AIDS epidemic, it was estimated that 740,000 people were living 
with HIV/AIDS in China [13]. Sexual contact has been found to be a major route of HIV transmission in China [13], and female sex workers (FSW) are at high risk of HIV infection. Based on data from 11 studies, FSW were more likely to be infected by HIV (3.0\%) compared with in all other women of reproductive age $(0.06 \%)$, with an odds ratio of 50.0 (95\% CI 46.0-54.4) [14]. FSW bear a disproportionate burden of HIV in China, accounting for almost half (48.6\%) of all HIV cases among women in that country [14]. Yet they continue to be excluded, sometimes systematically, from HIV services because of HIV/AIDS related stigma, discrimination, and criminalization.

In the past two decades, market-oriented reforms have radically changed the social landscape of China. One of the most significant developments has been the unprecedented growth in transient rural to urban migration. There have been more than 230 million migrants, approximately $17 \%$ of the total population in China. Uniquely, FSW are a marginalized sub-group of women as well as a marginalized sub-group of migrants [15]. Due to their limited education and skills, these women have few job opportunities. Unable to find a source of income in the cities, some women engage in commercial sex work to earn a living, thus becoming vulnerable to contracting HIV [15-19]. Many of the HIV-infected individuals in China are believed to be among the nation's 230 million rural-to-urban migrants. Correspondingly, they could have played important roles in HIV transmission in China.

There are several knowledge gaps in the existing literature regarding HIV related stigma. First, few studies have examined the HIV related stigma among FSW. Second, most of the previous studies were conducted among HIV positive individuals, and the HIV related stigma among FSW has not been well documented. Although the majority of FSW are migrants, the differences regarding HIV/AIDS related stigma between FSW and general migrant women (GMW) have not been well explored. To date, no study has yet targeted two kinds of subpopulations together. Interestingly, the two types of subjects share some characteristics, such as frequent moving, unstable work or away from family. Furthermore, these two types of workers are loosely organized and offering HIV education programs to them is understandably difficult. Therefore, in the present study, we compared HIV related stigma and HIV knowledge between FSW and GMW, and examined factors affecting HIV related stigma.

\section{Methods}

\section{Study setting and participants}

The present study was conducted during the period from September 2011 to December 2012 in Minhang District, Shanghai, one of the most developed metropolises in
China. The first HIV case in Shanghai was reported in 1987. By the end of 2012, 7,647 HIV/AIDS cases had been reported, $90 \%$ of the newly identified cases were sexually transmitted (Shanghai CDC HIV/STDs surveillance data, 2012).

For purposes of this study, a migrant woman was defined as someone who was born and registered as a permanent resident in a rural area outside of Shanghai but was living in Shanghai at the time when the survey was conducted. A female sex worker was a woman who has regularly exchanged sex services for money in commercial establishments such as karaoke bars or nightclubs for more than 6 months.

Thus far, two groups of women were recruited into the current study. Female sex workers were those who: 1) were aged between 18 and 65 years; 2) had been conducting commercial sex activities; 3 ) had been living in the community for more than 3 months; 4) had no official status of local residency; and 5) signed an informed consent. General migrant women were those who: 1 ) were aged between 18 and 65 years; 2) had been living in the community for more than 3 months; 3 ) had no official status of local residency; and 4) signed an informed consent. All the participants were confirmed to be HIV negative by laboratory tests.

\section{Procedures}

A multiple stage cluster sampling method was applied to recruit the participants. In the first stage, three out fourteen communities were randomly selected. Because commercial sex is still illegal in China, the majority of FSWs work in entertainment venues or personal service sectors such as karaoke bars, nightclubs, and massage parlors. To obtain FSW participants for the study, venues such as karaoke bars and massage parlors that were known by the local public health agency to provide commercial sex services were listed and selected for sampling. Female sex workers at the selected venues were approached and asked to participate in the study. The procedures for selecting GMW as participants were different. A variety of venues (e.g. factories, markets) that employed GMW were selected. GMW working in these places were enrolled in the study. In general, sample selection gave priority to small-to-medium-sized establishments to prevent over representation by large ones in the given sample size. Research staff then made a visit to all the selected venues and approached all potential subjects individually, explaining the purpose of the study and how the information collected would be used. Initially, A total of 1,500 subjects, including $750 \mathrm{FSW}$ and $750 \mathrm{GMW}$, were approached, and 1,396 (93.1\%) agreed to participate in the survey.

Anonymous interviews were administered in private rooms. Based on a pilot qualitative study, the face-to-face 
interviews were preferred by participants. All the eligible individuals included in the study were interviewed by trained female physicians or female post-graduate students who had previous experience of administering epidemiological questionnaires. Interviewers were trained in the conceptual framework of the study, technical aspects of its implementation, and the ethical parameters involved in social research.

Ethical approval for the study was issued by the Ethics Committee of the School of Public Health, Fudan University. The nature and purpose of the study were explained to each participant, after which they were asked to sign their consent to participate in the survey. A copy of the signed consent form was given to the participant. All participants received cash incentives (US\$8) for participating and travel expense reimbursements.

\section{Measures}

The instrument design was guided by a literature review, key informants, and input from experts. The questionnaire was pilot-tested and refined prior to use, and was administered to collect information on demographic characteristics, knowledge of HIV/AIDS, HIV related stigma and perceptions of the availability of HIV/AIDS and STD related information and services etc.

Participants' prejudicial attitudes toward PLWHA were assessed by asking participants their level of agreement with statements such as "A person with HIV must have done something wrong and deserves to be punished", or "A person who has HIV is dirty". A total of 12 statements were used, and responses were coded on a 1 (strongly disagree) to 5 (strongly agree) scale (Cronbach's alpha $=0.80$ ).

Stigma is a complex issue that has its roots in many domains, including culture, economics, knowledge, and policy. Based on some highly relevant literatures [20-24], HIV related stigma was thought to be derived from socio-cultural values, morals, and intrapersonal emotions such as negative feelings, beliefs, and attitudes regarding PLWHA. These items, as presented in the structured questionnaire used in the study, were classified into four conceptual domains (see Additional file 1: Table A1). We believe these four domains are practically useful for evaluating the HIV related stigma among the study participants, at both the individual level and at the community level. The four conceptual domains are as follows:

(1) Community or personal norms towards PLWHA

(2) Blame for PLWHA

(3) Sympathy and support to PLWHA

(4) Fear of transmission and disease

The HIV-related stigma score was constructed by taking the sum of all 12 items, therefore, summary scores could range from 12 to 60 (highest degree of stigma).
The direction of some items was reversed so that a higher score consistently indicated a higher degree of general prejudicial attitude. The stigma score was analyzed as an ordinal response with three levels: low (score $\leq 30)$, medium $(30<$ score $\leq 45)$, and high (score $>45)$.

Knowledge of HIV/AIDS was assessed with 12 questions that have been used previously, together or separately, in many HIV studies to measure HIV-related knowledge [25-27]. With regard these questions, six questions were about the three transmission modes of HIV and condom use for HIV prevention, three regarding misconceptions about HIV transmission through mosquito bites, shaking hands and eating with an HIV-infected individual, two about HIV diagnosis and antibiotic usage, and one on whether HIV/AIDS was curable. For each item, a correct answer was given 1 point; stating "don't know" and incorrect answer received 0 points. Total scores could therefore range from 0 to 12 (Cronbach's alpha $=0.78$ ).

\section{Statistical analysis}

Data from the questionnaires were recorded in duplicate using EpiData 3.0 for Windows (The EpiData Association, Odense, Denmark). Data validation tools were also used to validate the duplicate data entry. The database was then transferred into Statistical Package for Social Science (SPSS) software 20.0 (SPSS Inc., Chicago, IL) for statistical analysis. Differences for demographic variables were tested using chi-square and t-test for categorical and continuous variables, respectively. To describe the response pattern of each individual HIV-related stigma item, the frequencies of each question were tabulated by groups. The difference in the HIV-related stigma scores between FSW and GMW groups was examined graphically by using boxplot. A boxplot is a convenient way of graphically depicting groups of numerical data through summaries of stigma scores. Multiple ordinal logistic regression was conducted to determine factors associated with increasing level of HIV related stigma. Odds ratios (ORs) and 95\% confidence intervals (CIs) were calculated. All statistical tests were two sided, and a $p$ value less than 0.05 was considered statistically significant.

\section{Results}

\section{Participant characteristics}

A total of 1,396 subjects, including 721 female sex workers and 675 general migrant women, were finally recruited for the present study. Demographic characteristics of the participants are shown in Table 1. Among the FSW, the mean age of all participants was $26.5 \pm 7.08$ years. They were predominantly Han ethnicity (94.5\%), 56.0\% of them were aged $18-25$ years, $57.3 \%$ were single or widowed, $23.7 \%$ had a high school education or above, and $19.8 \%$ of them reported having monthly income more than 4000 Yuan. Approximately, $51.2 \%$ of them had lived in Shanghai for 
Table 1 Socio-demographic characteristics of study participants: 721 female sex workers (FSW) and 675 general migrant women (GMW), Minhang District, Shanghai, China, 2011-2012

\begin{tabular}{|c|c|c|c|}
\hline \multirow[t]{2}{*}{ Characteristics } & FSW $(n=721)$ & GMW $(n=675)$ & Total $(n=1396)$ \\
\hline & $\mathrm{N}(\%)$ & $\mathrm{N}(\%)$ & $\mathrm{N}(\%)$ \\
\hline \multicolumn{4}{|l|}{ Age group $(p<0.001)$} \\
\hline $18-25$ years & $404(56.0)$ & $295(43.7)$ & $699(50.1)$ \\
\hline $26-35$ years & $229(31.8)$ & $194(28.7)$ & $423(30.3)$ \\
\hline$\geq 36$ years & $88(12.2)$ & $186(27.6)$ & $274(19.6)$ \\
\hline \multicolumn{4}{|l|}{ Ethnicity $(p=0.176)$} \\
\hline Han & $681(94.5)$ & $648(96.0)$ & $1329(95.2)$ \\
\hline Other & $40(5.5)$ & $27(4.0)$ & $67(4.8)$ \\
\hline \multicolumn{4}{|c|}{ Duration in Shanghai $(p=0.019)$} \\
\hline$<1$ year & $369(51.2)$ & $303(44.9)$ & $672(48.1)$ \\
\hline$\geq 1$ year & $352(48.8)$ & $372(55.1)$ & $724(51.9)$ \\
\hline \multicolumn{4}{|l|}{ Marital status $(p<0.001)$} \\
\hline Single/widowed & $429(57.3)$ & $242(35.9)$ & $671(48.1)$ \\
\hline Married & $292(42.7)$ & $433(64.1)$ & $725(51.9)$ \\
\hline \multicolumn{4}{|l|}{ Education $(p<0.001)$} \\
\hline Elementary school or lower & $128(17.8)$ & 85 (12.6) & $213(15.3)$ \\
\hline Middle school & $422(58.5)$ & $356(52.7)$ & $778(55.7)$ \\
\hline High school or higher & $171(23.7)$ & $234(34.7)$ & $405(29.0)$ \\
\hline \multicolumn{4}{|c|}{ Monthly income (Yuan, $p<0.001$ ) } \\
\hline$\leq 2000$ & 120 (16.6) & 389 (57.6) & $509(36.5)$ \\
\hline $2001-4000$ & $458(63.6)$ & $276(40.9)$ & $734(52.6)$ \\
\hline$\geq 4001$ & 143 (19.8) & $10(1.5)$ & 153 (10.9) \\
\hline
\end{tabular}

Abbreviations: FSW female sex workers, GMW general migrant women.

Chi-square test was applied for the comparison of these characteristics; P values less than 0.05 were considered statistically significant.

less than one year and $46.7 \%$ had migrated to more than 2 cities. Among the GMW, participants had a mean age of $29.4 \pm 8.4$ years, $43.7 \%$ of them were aged $18-25$ years, a majority (96.0\%) of them were Han ethnicity, $64.1 \%$ were married, $34.7 \%$ had a high school education or above, and very few (1.5\%) reported monthly income more than 4000 Yuan. More than half of them had lived in Shanghai for less than one year and nearly half of them had migrated to more than 2 cities.

Compared with the GMW group, FSW were significantly younger $(26.5 \pm 7.08$ years vs. $29.4 \pm 8.4$ years, $\mathrm{P}<0.001$ ), and were more likely to have a shorter duration in Shanghai, and to be unmarried and less educated. Likewise, FSW were more likely to earn a higher income. There was no significant difference in ethnicity between the two groups.

\section{HIV/AIDS related knowledge}

Scores on HIV/AIDS related knowledge ranged from 0 to 12. Both FSW and GMW had a substantial misconception regarding HIV transmission, prevention, diagnosis and treatment, and FSW had a slightly but significantly higher score than GMW $(8.21 \pm 2.8$ vs. $7.64 \pm 2.8, \mathrm{p}<0.001)$. The most serious misconceptions concerned the transmission possibility of mosquito bites and the use of condoms for HIV prevention. Approximately $34.3 \%$ of FSW and $37.5 \%$ GMW incorrectly reported that HIV could be transmitted via mosquito bites. At the same time, only $70.5 \%$ of FSW and $54.4 \%$ GMW correctly reported that condom use can prevent HIV transmission.

\section{HIV related stigma}

Both groups of women displayed a medium level of HIV related stigma. The mean of general prejudicial attitudes was $38.34 \pm 6.21$ and $38.35 \pm 6.86$ for FSW and GMW, respectively. The summed scores ranged from 20 to 50 among FSW and from 19 to 50 among GMW. No difference was detected between the FSW and GMW groups in the stigma scores (Figure 1). The stigma was further categorized into four domains: Domain (1) mean score $9.38 \pm 2.08 v s .9 .33 \pm 1.99$ for FSW and GMW, $\mathrm{p}=0.652$, Domain (2) mean score $12.39 \pm 2.8$ vs. $12.94 \pm 2.91$ for FSW and GMW, p $<0.001$, (3) mean score $5.98 \pm 1.34$ vs. $5.77 \pm 1.47$ for FSW and GMW, $\mathrm{p}=0.05$ (4) mean 


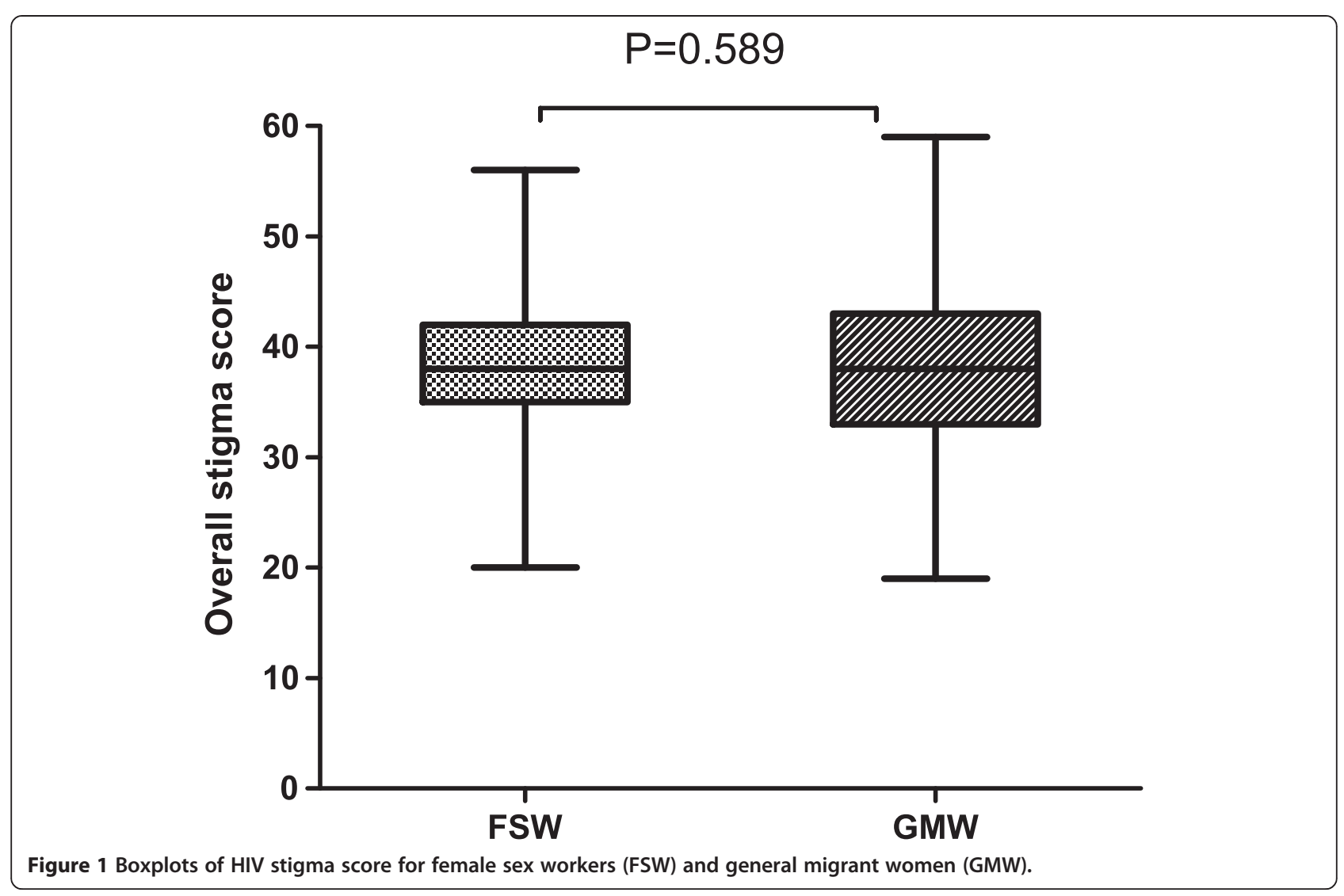

score $10.58 \pm 2.31$ vs. $10.57 \pm 2.28$ for FSW and GMW, $\mathrm{p}=0.903$.

Table 2 shows the results from descriptive analyses of HIV related stigma measures by four domains. A significant difference between FSW and GMW was observed for the statements such as "People who get HIV/AIDS through sex or drug use get what they deserve" and "AIDS is a punishment for bad behavior". Similar patterns were observed with the statements that measured the stigmatizing attitudes. Conversely, no significant difference was detected between the two groups in other statements such as, "People living with HIV should have the right to marry" and "You are afraid of people living with HIV". Overall, HIV related stigma was prevalent in both groups, and a large proportion of the study participants were afraid of people living with HIV.

\section{Correlates of HIV related stigma among study participants}

An ordinal logistic regression was used to determine the factors associated with stigma scores (low (score $\leq 30$ ), medium $(30<$ score $\leq 45)$, and high (score $>45)$ ) (Table 3 ). With regard to the FSW group, results indicated that those who were in the older age group were more likely to report prejudicial attitude (age 26-35 years, OR, 2.06, 95\% CI 1.06-4.01). Those living in Shanghai for a year or more were more likely to report negative attitudes than those who had lived in Shanghai less than 1 year (OR, 1.37, 95\% CI 0.96-1.97), although the association was marginal. In addition, married FSW were more likely to manifest a higher level of stigma than those who were single (OR, 1.62, 95\% CI 1.03-2.54). Moreover, those who working in some lower level sex service sites were more likely to show stigma attitude (OR, 1.60, 95\% CI 1.06-2.43). Conversely, HIV knowledge was inversely associated with HIV related stigma level (OR, 0.93, 95\% CI 0.87-0.98).

Results for GMW indicated that those in the older age group (age 26-35 years) were more likely to express a higher level of stigma (OR, 2.61, 95\% CI 1.03-2.54), and HIV knowledge was found to be inversely associated with the level of HIV related stigma as well (OR, 0.89, 95\% CI 0.84-0.95).

\section{Discussion}

HIV/AIDS related stigma has been a barrier to HIV/AIDS testing, treatment and care, since the beginning of the HIV/AIDS epidemic in China 25 years ago [4,5,26,28,29]. The differences in HIV related stigma between FSW and GMW have not been previously investigated in China. Information from the present study may benefit programs targeting reduction of HIV/AIDS stigma among FSW and GMW in this country. 
Table 2 Comparisons of HIV related stigma measures between 721 female sex workers and 675 general migrant women in Shanghai from 2011-2012

\begin{tabular}{lll}
\hline Domain & FSW & GMW \\
$(n=721)$ & $(n=675)$ \\
& $N(\%)$ & $N(\%)$ \\
\hline
\end{tabular}

Domain One: Community or personal norms towards people living with HIV

You would feel ashamed if someone you know got HIV/AIDS $(p=0.008)$

Agree

Not sure

$256(35.5)-203(30.1)$

Disagree

You would feel ashamed if someone in your family got HIV/AIDS $(p=0.161)$

Agree

Not sure

Disagree

You would be willing to work with an HIV-positive coworker $(p=0.499)$

Agree

Not sure

Disagree

Domain Two: Blame for people living with HIV

AIDS is a punishment for bad behavior $(p=0.015)$

Agree

Not sure

Disagree

People who behave promiscuously should be blamed for AIDS $(p<0.001)$

Agree

Not sure

Disagree

People who get HIV/AIDS through sex or drug use, get what they deserve $(p<0.001)$

Agree

Not sure

Disagree

People who have HIV are dirty $(p<0.001)$

Agree

Not sure

Disagree

Domain Three: Sympathy to people living with HIV

People living with HIV should have the right to marry $(p=0.072)$

Agree

Not sure

Disagree
$345(42.0) \quad 271(40.1)$

$274(33.4) \quad 203(30.1)$

$\begin{array}{ll}227(31.5) & 196(29.0) \\ 238(33.0) & 276(40.9)\end{array}$

$285(39.5) \quad 240(35.6)$

$188(26.1) \quad 171(25.3)$

248 (34.4) 264 (39.1)

$173(24.0) \quad 174(25.8)$

$217(30.1) \quad 212(31.4)$

$331(45.9) \quad 289(42.8)$

242 (33.6) 263 (38.9)

$267(37.0) \quad 202(30.0)$

$212(29.4) \quad 210(31.1)$

$301(41.7) \quad 457(67.7)$

$263(36.5) \quad 124(18.4)$

$157(21.8) \quad 94(13.9)$

$262(36.3) \quad 312(46.2)$

$263(36.5) \quad 199(29.5)$

$196(27.2) \quad 164(24.3)$

202 (28.1) 189 (28.0)

$306(42.4) \quad 208(30.8)$

$213(29.5) \quad 278(41.2)$

$202(24.6) \quad 201(29.8)$
Table 2 Comparisons of HIV related stigma measures between 721 female sex workers and $\mathbf{6 7 5}$ general migrant women in Shanghai from 2011-2012 (Continued)

People with HIV should lose their jobs

$(p=0.006)$

Agree

$226(31.3) \quad 178(26.4)$

Not sure

$287(39.8) \quad 249(36.9)$

Disagree

$208(28.9) \quad 248(36.7)$

Domain Four: Fear of transmission

and disease

You are afraid of people living with HIV/AIDS $(p=0.098)$

Agree

$495(68.7) \quad 432(64.0)$

Not sure

$148(20.5) \quad 147(21.8)$

Disagree

$78(10.8) \quad 96(14.2)$

You would not buy anything from a

food vendor who has HIV/AIDS

$(p=0.127)$

Agree

$379(52.6) \quad 387(57.3)$

Not sure

$184(25.5) \quad 166(24.6)$

Disagree

$158(21.9) \quad 122(18.1)$

You would not share eating utensils with a person living with HIV because you are afraid of getting infected $(p=0.836)$

$\begin{array}{lll}\text { Agree } & 418(57.9) & 382(56.6) \\ \text { Not sure } & 166(23.1) & 157(23.2) \\ \text { Disagree } & 137(19.0) & 136(20.1)\end{array}$

Abbreviations: FSW female sex workers, GMW general migrant women.

The Chi-square test was applied for the comparison of these characteristics, $p$ values less than 0.05 were considered statistically significant.

The current study identified a medium level of HIV/ AIDS stigma in both groups of participants. These findings are consistent with previous studies [30-33], and indicate that negative feelings may be widespread among various populations towards someone with HIV, suggesting that this is an important public health issue that needs to be urgently addressed. Our finding that approximately $70 \%$ of the participants were afraid of HIV infected individuals strongly suggests an urgent need to initiate health education programs to reduce discrimination towards PLWHA. Interestingly, FSW showed a somewhat lower level of HIV/AIDS related stigma in specific stigma items compared with GMW, although no significant differences were detected with regard to the summary scores of HIV related stigma. A better acceptability among FSW was noted in responses to some of the questions. For example, regarding the statement, "People who get HIV/AIDS through sex or drug use get what they deserve", only $36.3 \%$ of FSW said "yes", compared with $46.2 \%$ of GMW; likewise, for the statement, "People who behave promiscuously should be blamed 
Table 3 Potential risk factors for HIV related stigma among female sex workers $(n=721)$ and general migrant women $(n=675)$

\begin{tabular}{|c|c|c|c|c|c|c|}
\hline \multirow[t]{2}{*}{ Variables } & \multicolumn{3}{|c|}{ FSW $(n=721)$} & \multicolumn{3}{|c|}{ GMW (n = 675) } \\
\hline & OR & $95 \% \mathrm{Cl}$ & P-value & OR & $95 \% \mathrm{Cl}$ & P-value \\
\hline HIV knowledge & 0.93 & $0.87-0.98$ & 0.019 & 0.89 & $0.84-0.95$ & $<0.001$ \\
\hline \multicolumn{7}{|l|}{ Age (years) } \\
\hline $18-25$ & Ref & - & - & Ref & - & - \\
\hline $26-35$ & 2.06 & $1.06-4.01$ & 0.033 & 2.61 & $1.49-4.55$ & 0.001 \\
\hline$\geq 36$ & 1.06 & $0.57-1.98$ & 0.853 & 1.53 & $0.96-2.45$ & 0.074 \\
\hline \multicolumn{7}{|l|}{ Education level } \\
\hline Elementary or low & Ref & - & - & Ref & - & - \\
\hline Middle school & 0.15 & $0.46-1.51$ & 0.531 & 0.85 & $0.45-1.60$ & 0.604 \\
\hline High school or higher & 1.08 & $0.69-1.68$ & 0.731 & 0.83 & $0.56-1.24$ & 0.361 \\
\hline \multicolumn{7}{|l|}{ Ethnicity } \\
\hline Han & Ref & - & - & Ref & - & - \\
\hline Minority & 1.38 & $0.66-2.99$ & 0.375 & 1.46 & $0.63-3.39$ & 0.379 \\
\hline \multicolumn{7}{|l|}{ Marital status } \\
\hline Single/widowed & Ref & - & - & Ref & - & - \\
\hline Married & 1.62 & $1.03-2.54$ & 0.036 & 1.64 & $0.82-3.12$ & 0.160 \\
\hline \multicolumn{7}{|c|}{ Living with spouse or a sexual partner } \\
\hline Yes & Ref & - & - & Ref & - & - \\
\hline No & 0.92 & $0.62-1.36$ & 0.686 & 1.25 & $0.70-2.25$ & 0.453 \\
\hline \multicolumn{7}{|l|}{ Perceived HIV risk } \\
\hline Yes & Ref & - & - & Ref & - & - \\
\hline No & 0.75 & $0.38-1.48$ & 0.403 & 0.73 & $0.34-4.56$ & 0.413 \\
\hline \multicolumn{7}{|l|}{ Heard of VCT } \\
\hline Yes & Ref & - & - & Ref & - & - \\
\hline No & 0.16 & $0.54-1.28$ & 0.407 & 1.25 & $0.85-1.97$ & 0.224 \\
\hline \multicolumn{7}{|c|}{ Monthly income (Yuan) } \\
\hline$\leq 2000$ & Ref & - & - & Ref & - & - \\
\hline $2001-4000$ & 0.91 & $0.47-1.93$ & 0.777 & 0.74 & $0.12-3.01$ & 0.672 \\
\hline$\geq 4001$ & 1.48 & $0.91-2.43$ & 0.114 & 0.57 & $0.14-2.31$ & 0.431 \\
\hline \multicolumn{7}{|l|}{ Duration in Shanghai } \\
\hline$<1$ year & Ref & - & - & Ref & - & - \\
\hline$\geq 1$ year & 1.37 & $0.96-1.97$ & 0.086 & 1.23 & $0.87-1.73$ & 0.235 \\
\hline \multicolumn{7}{|l|}{ Having tested HIV } \\
\hline Yes & Ref & - & - & Ref & - & - \\
\hline No & 1.08 & $0.63-1.84$ & 0.780 & 0.98 & $0.17-5.77$ & 0.998 \\
\hline \multicolumn{7}{|l|}{ Sex working sites } \\
\hline High level & Ref & - & - & N/A & $\mathrm{N} / \mathrm{A}$ & N/A \\
\hline Low level & 1.60 & $1.06-2.43$ & 0.026 & N/A & N/A & N/A \\
\hline
\end{tabular}

Abbreviations: FSW female sex workers, GMW general migrant women.

Ordinal logistic regression analysis was performed. $\mathrm{OR}=$ odds ratio, $\mathrm{Cl}=$ confidence interval, $\mathrm{p}$-values less than 0.05 were considered statistically significant.

for AIDS", the proportion of FSW agreeing was 41.7\%, compared with $67.7 \%$ for GMW. These results are in accordance with those from a previous study $[8,34]$.
The present study showed that HIV/AIDS knowledge was inversely associated with HIV/AIDS related prejudicial attitudes, which are consistent with the results from 
previous studies $[8,25,35]$. Thus far, the HIV/AIDS knowledge may cast an impact on the level of HIV/AIDS related stigma [36]. Of note, despite increasing concern about HIV/AIDS among public health officials in China, widespread misconceptions exist among both groups about HIV transmission [37,38]. Similarly, substantial misconceptions regarding HIV/AIDS knowledge were detected among study participants in the present study. The co-existence of correct and incorrect knowledge has been documented in other settings and may reflect preexisting misunderstandings of disease transmission combined with more recent exposure to HIV prevention information $[39,40]$. Moreover, a slightly higher HIV/AIDS knowledge level was detected among FSW compared with GMW. Based on these findings, stigma reduction intervention programs should consider giving a priority to the delivery of HIV/AIDS knowledge to both FSW and GMW, which might help reduce HIV related stigma.

The present study also showed that older women were more likely to report a negative attitude. This result is consistent with previous studies that less stigma was reported among person under 30 years of age $[8,22,41,42]$. It suggests that older women were more likely to hold negative attitude towards PLWHA. In the current study, women who had been living in Shanghai longer and women who were married were more likely to show a stigma attitude. These women may have a more stable living situation, compared with those who have lived in Shanghai for shorter periods, and therefore may have higher economic status. Data have shown that more than half of the participants, in either the FSW or GMW group, migrated frequently across different cities. Their migrant status is also of importance in the HIV/AIDS prevention program, which deserves extensive research. Therefore, HIV/AIDS stigma reduction programs should be specifically designed by taking these factors into consideration. Moreover, those FSW from lower level sex service site were more likely to report higher HIV-related stigma. It indicates that stigma reduction interventions among these specific groups are highly warranted.

This study has allowed us to address previously unexplored questions in the context of a relatively large, representative population. However, the study has several limitations. First, due to the observational nature of the data, it could limit causal inference ability. Second, selfreported data are subject to self-reporting bias. Female sex workers whose activities are still regarded as illegal in China may provide a socially desirable answer, which may underestimate the stigma attitude. Third, we analyzed the potential correlates for HIV related stigma based on prior knowledge and the available literature, which may not comprehensive. Finally, respondents were recruited from a relatively prosperous metropolitan area in China. This aspect of the sample may restrict its generalizability; however, it is likely that the characteristics of the study participants reflect the situation in similar settings in China.

The present study provides new evidence that HIV/ AIDS related stigma is prevalent among both FSW and GMW in Shanghai, China. HIV/AIDS-related public health interventions are needed to facilitate reduction and eventual elimination of HIV/AIDS-related stigma in both populations.

\section{Conclusions}

The present study suggests an urgent need for the development of appropriate education strategies to reduce HIV related stigma for women, particularly those who are older, less educated, and have lived in Shanghai relatively longer. Both FSW and GMW should be targeted in future stigma reduction programs.

\section{Additional file}

\section{Additional file 1: Table A1. Selected Conceptual Domains and}

Questions/Probes in the Structured Interview Guide.

\section{Competing interests}

The authors declare that they have no competing interests.

\section{Authors' contributions}

$\mathrm{TZ}$ conceived the idea for the study. $Y Y, J W, F L, T Z, F Y$, and $Y Z$ obtained and managed the data. JW, FL, YY, and TZ (Tao Zhang) participated in the study implementation, data analysis and interpretation. YY and TZ (Tao Zhang) cleaned the data and completed the statistical analysis. YY and TZ drafted the manuscript, which was reviewed by all authors. All authors read and approved the final manuscript.

\section{Acknowledgments}

We would like to thank all participants for their attending into this study. We are also grateful to staff from local community health service centers for their assistance. This study was supported by Fundamental Research Funds for the Central Universities (10FX058 to TZ), Supported by Doctoral Fund of Ministry of Education of China (20120071120050, 20520133104 to TZ), and the Shanghai Leading Disciplinary Project (grant no. 12GWZX1010).

\section{Author details}

${ }^{1}$ Department of Epidemiology, School of Public Health, Fudan University, Shanghai, China and Key Laboratory of Public Health Safety (Fudan University), Ministry of Education, Shanghai, China. ${ }^{2}$ Minhang District Center for Diseases Control and Prevention, Shanghai, China.

Received: 11 February 2014 Accepted: 17 December 2014 Published online: 22 January 2015

\section{References}

1. Herek GM, Capitanio CJ. AIDS stigma and contact with persons with AIDS: effects of direct and vicarious contact. J Appl Soc Psych. 1997;27:1-36.

2. Mahajan AP, Sayles JN, Patel VA, Remien RH, Sawires SR, Ortiz DJ, et al. Stigma in the HIV/AIDS epidemic: a review of the literature and recommendations for the way forward. AIDS (London, England). 2008;22 Suppl 2:S67-79.

3. Parker R, Aggleton P. HIV and AIDS-related stigma and discrimination: a conceptual framework and implications for action. Soc Sci Med. 2003;57(1):13-24.

4. Lieber E, Li L, Wu Z, Rotheram-Borus MJ, Guan J, National Institute of Mental Health Collaborative HIVPTG. HIV/STD stigmatization fears as health-seeking barriers in China. AIDS Behav. 2006;10(5):463-71. 
5. Sullivan SG, Xu J, Feng $Y$, Su S, Xu C, Ding $X$, et al. Stigmatizing attitudes and behaviors toward PLHA in rural China. AIDS Care. 2010;22(1):104-11.

6. Wu Z, Sullivan SG, Wang Y, Rotheram-Borus MJ, Detels R. Evolution of China's response to HIV/AIDS. Lancet. 2007:369(9562):679-90.

7. Li L, Liang LJ, Lin C, Wu Z, Wen Y. Individual attitudes and perceived social norms: Reports on HIV/AIDS-related stigma among service providers in China. Int J Psych. 2009;44(6):443-50.

8. Cao H, He N, Jiang Q, Yang M, Liu Z, Gao M, et al. Stigma against HIV-infected persons among migrant women living in Shanghai, China. AIDS Educ Prev. 2010:22(5):445-54.

9. Burki TK. Discrimination against people with HIV persists in China. Lancet. 2011;377(9762):286-7.

10. Qiu J. China. Stigma of HIV imperils hard-won strides in saving lives. Science (New York, NY). 2011;332(6035):1253-4.

11. Wang N, Wang L, Wu Z, Guo W, Sun X, Poundstone K, et al. Estimating the number of people living with HIV/AIDS in China: 2003-09. Int J Epidemiol. 2010;39(2):ii21-8

12. Wu Z, Rou K, Cui H. The HIV/AIDS epidemic in China: history, current strategies and future challenges. AIDS Educ Prev. 2004;16(3):7-17.

13. The Estimation of HIV/AIDS in China in 2009

14. Baral S, Beyrer C, Muessig K, Poteat T, Wirtz AL, Decker MR, et al. Burden of HIV among female sex workers in low-income and middle-income countries: a systematic review and meta-analysis. Lancet Infect Dis. 2012;12(7):538-49.

15. Hong $Y$, Li X. Behavioral studies of female sex workers in China: a literature review and recommendation for future research. AIDS and behavior. 2008:12(4):623-36

16. He N, Detels R, Chen Z, Jiang Q, Zhu J, Dai Y, et al. Sexual behavior among employed male rural migrants in Shanghai. China AIDS Educ Prev. 2006;18 (2):176-86

17. Li X, Fang $X$, Lin D, Mao R, Wang J, Cottrell L, et al. HIV/STD risk behaviors and perceptions among rural-to-urban migrants in China. AIDS Educ Prev. 2004;16(6):538-56

18. Liu H, Li X, Stanton B, Liang G, Chen X, Yang H, et al. Risk factors for sexually transmitted disease among rural-to-urban migrants in China: implications for HIV/sexually transmitted disease prevention. AIDS Patient Care STDS. 2005;19(1):49-57.

19. Qian HZ, Vermund SH, Wang N. Risk of HIV/AIDS in China: subpopulations of special importance. Sex Transm Infect. 2005;81(6):442-7.

20. Zhang C, Hong Y, Li X, Qiao S, Zhou Y, Su S: Psychological Stressors in the Context of Commercial Sex Among Female Sex Workers in China. Health Care Women Int 2013

21. Zhao Q, Li X, Zhao G, Zhao J, Fang X, Lin X, et al. AIDS knowledge and HIV stigma among children affected by HIV/AIDS in rural China. AIDS Educ Prev. 2011;23(4):341-50

22. Vlassoff C, Weiss MG, Rao S. A question module for assessing community stigma towards HIV in rural India. J Biosocial Sci. 2013;45(3):359-74.

23. Li L, Liang LJ, Lin C, Wu Z, Rotheram-Borus MJ: HIV prevention intervention to reduce HIV-related stigma: evidence from China. AIDS (London, England) 2010, 24(1):115-122

24. Qiao S, Li X, Zhang C, Zhou Y, Shen Z, Tang Z, et al. Psychological Fears among Low-Paid Female Sex Workers in Southwest China and Their Implications for HIV Prevention. PLoS One. 2014;9(10):e111012.

25. Chen J, Choe MK, Chen S, Zhang S. Community environment and HIV/AIDS-related stigma in China. AIDS Educ Prev. 2005;17(1):1-11.

26. Li L, Lin C, Wu Z, Wu S, Rotheram-Borus MJ, Detels R, et al. Stigmatization and shame: consequences of caring for HIV/AIDS patients in China. AIDS Care. 2007;19(2):258-63.

27. Carey MP, Schroder KE. Development and psychometric evaluation of the brief HIV Knowledge Questionnaire. AIDS Educ Prev. 2002;14(2):172-82.

28. Li L, Wu Z, Wu S, Zhaoc Y, Jia M, Yan Z. HIV-related stigma in health care settings: a survey of service providers in China. AIDS Patient Care STDS. 2007;21(10):753-62.

29. Hong Y, Li X, Stanton B, Fang X, Lin D, Wang J, et al. Expressions of HIV-related stigma among rural-to-urban migrants in China. AIDS Patient Care STDS. 2008:22(10):823-31.

30. Hong $Y$, Fang $X$, Li X, Liu Y, Li M, Tai-Seale T. Self-perceived stigma, depressive symptoms, and suicidal behaviors among female sex workers in China. J Transcultural Nurs. 2010;21(1):29-34
31. Ugarte WJ, Hogberg U, Valladares EC, Essen B. Measuring HIV- and AIDS-related stigma and discrimination in Nicaragua: results from a community-based study. AIDS Educ Prev. 2013;25(2):164-78.

32. Getnet MK, Damen HM. Level of stigma among female sex workers: comparison of two surveys of HIV behavioral data, Ethiopia. African Health Sci. 2011;11(4):543-9.

33. Tenkorang EY, Owusu AY: Examining HIV-related stigma and discrimination in Ghana: what are the major contributors? Sexual Health 2013, 10(3):253-262

34. Hesketh T, Zhang J, Qiang DJ. HIV knowledge and risk behaviour of female sex workers in Yunnan Province, China: potential as bridging groups to the general population. AIDS Care. 2005;17(8):958-66.

35. Kalichman SC, Simbayi LC, Cain D, Jooste S, Skinner D, Cherry C Generalizing a model of health behaviour change and AIDS stigma for use with sexually transmitted infection clinic patients in Cape Town, South Africa. AIDS Care. 2006;18(3):178-82

36. Robertson AM, Ojeda VD, Nguyen L, Lozada R, Martinez GA, Strathdee SA et al. Reducing harm from HIV/AIDS misconceptions among female sex workers in Tijuana and Ciudad Juarez, Mexico: A cross sectional analysis. Harm Reduction J. 2012;9(1):35

37. Qian HZ, Wang N, Dong S, Chen H, Zhang Y, Chamot E, et al. Association of misconceptions about HIV transmission and discriminatory attitudes in rural China. AIDS Care. 2007;19(10):1283-7.

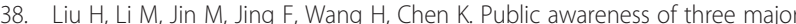
infectious diseases in rural Zhejiang province, China: a cross-sectional study. BMC Infect Dis. 2013;13(1):192.

39. London AS, Robles A. The co-occurrence of correct and incorrect HIV transmission knowledge and perceived risk for HIV among women of childbearing age in El Salvador. Soc Sci Med. 2000;51(8):1267-78.

40. Tsala Dimbuene Z, Kuate Defo B: Fostering accurate HIV/AIDS knowledge among unmarried youths in Cameroon: do family environment and peers matter? BMC Public Health 2011, 11:348

41. Jacobs RJ, Kane MN. HIV-related stigma in midlife and older women. Social Work Health Care. 2010;49(1):68-89.

42. Messer LC, Pence BW, Whetten K, Whetten R, Thielman N, O'Donnell K, et al. Prevalence and predictors of HIV-related stigma among institutional- and community-based caregivers of orphans and vulnerable children living in five less-wealthy countries. BMC Public Health. 2010;10:504.

\section{Submit your next manuscript to BioMed Central and take full advantage of:}

- Convenient online submission

- Thorough peer review

- No space constraints or color figure charges

- Immediate publication on acceptance

- Inclusion in PubMed, CAS, Scopus and Google Scholar

- Research which is freely available for redistribution

Submit your manuscript at www.biomedcentral.com/submit 